\title{
Singular Spectrum Analysis of Nonstationary Tidal Currents Applied to ADCP Data from the Northeast Brazilian Shelf
}

\author{
MARCIO L. VIANNA \\ Instituto Nacional de Pesquisas Espaciais, and VM Oceanica Ltda, Sao Jose dos Campos, Sao Paulo, Brazil \\ ViviAne V. MENEZES \\ VM Oceanica Ltda, Sao Jose dos Campos, Sao Paulo, Brazil
}

(Manuscript received 12 August 2004, in final form 5 April 2005)

\begin{abstract}
The development of new tools for the analysis of nonstationary currents, including tidal currents, has been the subject of recent research. In this work a method for studies of nonstationary barotropic or baroclinic currents based on empirical orthogonal function (EOF) and singular spectrum analysis (SSA) is proposed. It represents a new alternative to other methods of analysis of tidal currents in strong interaction with nontidal forcing, for example, the continuous wavelet transform. The advantage of the SSA method resides in the fact that it is fast, easy to implement, efficient for short-time records, and is based on the covariance structure of the data. If significant tidal constituents occur in the measurements, these are determined by the method itself even with short-time-series records. This is in contrast to the harmonic analysis (HA), where a large table of tidal constituents stated a priori are fitted to the data, even if the presence of some of these are spurious and not justified physically. The method is first demonstrated in the analysis of a synthetic current time series and then applied to an hourly current ADCP profile dataset of 410 days from the northeast Brazilian shelf. In both cases the SSA results were compared to the classical HA and the neoclassical short-term HA (STHA). The description of the shelf area where the ADCP was placed, the deployment and data acquisition operations, and the quality control data analysis are included for completeness. Analysis of the full ADCP quality-controlled data was done after a separation of the subtidal from the tidal high-frequency bands, although this traditional separation is not strictly necessary and was only made to better compare with HA and STHA. Analysis of the tidal band obtained from the ADCP data showed that the extracted tidal ellipse constituents present coherent oscillations dominated by the annual and 57-day periods, and changes in the sense of rotation of the current vector from anticyclonic to cyclonic in the ellipses. The subtidal band variability is shown to be also dominated by an annual and a 57-day period component, both polarized along the isobaths, which is suggestive of a nonlinear interaction of the subtidal and the tidal variability.
\end{abstract}

\section{Introduction and motivation}

The northeast Brazilian shelf is characterized by the presence of a rich suite of quartz sand bedforms having heights of 3-10 m (between 0.1 and 0.3 of the local water depth) and 100-4000-m crest lengths, which are clearly visible by satellite imagery up to $40-\mathrm{m}$ depth, as shown by Vianna et al. $(1991,1993)$ and Santos (1999).

Corresponding author address: M. Vianna, CIP/INPE, Instituto Nacional de Pesquisas Espaciais, Av. Dos Astronautas 1758, S. J. Campos, SP 12227-010, Brazil.

E-mail: marcio@vmoceanica.com.br
It is a very shallow shelf, with the edge having depths of the order of $50 \mathrm{~m}$, at $50-100-\mathrm{km}$ distances from the coast. The area is within the Potiguar basin, the second most important oil production basin in Brazil and, economically and socially, the most important lobster fishing ground. Moreover, the exquisite adjacent beach landscapes are associated with intense tourism activities throughout the whole year.

The impact of the kilometer-scale bedform fields, generated by currents and waves on a time scale of decades to centuries, strongly modifies ocean circulation patterns in the shallow shelf through bottom interactions. The importance of these varying circulation patterns for oil spill risk analysis has never been as- 
sessed, since it requires good data in support to realistic parameterizations to be used in modeling.

Quantitatively, this is still one of the less-studied shelves of the world, with very few good in situ datasets available; most of those in existence are proprietary in the oil industry, and even these do not span a full year. In terms of coastal sea level, all quality-controlled data from this shelf known to the authors are those from the ports of Luis Correia, Fortaleza, and Termisa Island, which are available both from the World Ocean Circulation Experiment database (WOCE 2002) and from the University of Hawaii Sea Level Center Web page (http://uhslc.soest.hawaii.edu). In terms of currents, to date only two studies have been reported in the open literature, one by Signorini and Miranda (1983) in the northeast Brazilian shelf and the other by the project team of A Multidisciplinary Amazon Shelf Sediment Study (AMASSEDS) in the Amazon shelf (Beardsley et al. 1995).

Signorini and Miranda (1983) based their work on a 40-day mooring with current meters at three depths: near the shelf break, at $3^{\circ} \mathrm{S}$, and at a local depth of 41 $\mathrm{m}$. They showed that the currents were dominated by cross-shore semidiurnal tidal currents and subtidal unidirectional alongshore flow of the same order of magnitude.

AMASSEDS was based mainly on data from an array of current meter moorings deployed between 1990 and 1991 at around $3^{\circ} \mathrm{N}$, focusing on the prevailing M2 tidal currents. The AMASSEDS project showed that the Amazon shelf is also dominated by complex bottom topography and composition, and strong river discharges, which were shown to perturb the expected first-order cross-isobath M2 tidal circulation.

However, no further studies have been published since 1983 on the Southern Hemisphere part of this shelf. To start closing this gap in knowledge, we initiated a long-term study by deploying south of $2^{\circ} \mathrm{S}$ two bottom-mounted RD Instruments (RDI) Workhorse ADCP moorings working in the self-contained mode, one at the 18-m isobath and another one near the shelf edge at 30-m depth. The data acquisition scheme was planned to consist of independent 3-month retrievals and redeployments, to complete one full year of data.

Preliminary studies of partial datasets obtained from the two moorings, using harmonic analysis (HA), suggested that the tidal ellipse constituents were not stationary: the direction of the major axis of the M2 tidal ellipses in one segment was offset by almost $10^{\circ}$ in relation to the other, well above the confidence intervals at the $95 \%$ level. When the complete 410 -day series was used, the result for the M2 major axis inclination was $\theta$ $=41.85^{\circ}$ and the confidence interval was $\varepsilon_{\theta}=1.75$, also
TABLE 1 . The M2 ellipse major axis inclination $\theta^{\circ}$ computed with HA.

\begin{tabular}{lcc}
\hline Time interval & Length (days) & $\theta\left({ }^{\circ}\right)$ \\
\hline 24 Sep 2000-2 Dec 2002 & 69 & $48.1 \pm 3.7$ \\
9 Mar 2001-17 May 2001 & 69 & $35.7 \pm 3.6$ \\
24 Sep 2000-24 Nov 2001 & 410 & $41.9 \pm 1.7$ \\
\hline
\end{tabular}

at the $95 \%$ level. No significant low-frequency component modulating the tidal ellipse constituents was found either in the unfiltered or in the high-pass-filtered vector signals. Table 1 summarizes these findings for the deeper mooring. Our conclusion was that the method of harmonic analysis did not seem to be adequate to this study.

By scanning over the recent literature, we found a nice work by Jay and Kukulka (2003), which presents a review of methods of analysis of tidal records containing multiscale nontidal modulations and suggests that their continuous wavelet transform (CWT) methodology offers the best results for such analysis.

The fact that tidal currents are multiply quasiperiodic nonstationary vector signals embedded in a nonstationary and noisy background suggests that the information contained in the covariance structure is indeed very important and should be taken into account. The possible deficiencies of CWT reside in the fact that it does not use this information, and in this sense CWT seems to constitute at most an improvement of Fouriertype analysis for nonstationary data. These look like the same deficiencies related to the classical technique of complex demodulation (CD) (Bloomfield 1976), which would be a first choice to separate lowerfrequency modulations from the higher-frequency carrier signal. As is the case in all Fourier-derived techniques, one needs filtering and/or tapering (window carpentry) as a side work, losing endpoints of the data series, in addition to the deficiencies noted by Jay and Kukulka (2003).

On the other hand, empirical orthogonal function (EOF) time domain analysis, as is the case of singular spectrum analysis (SSA), is based on data covariances, so it can potentially be a more naturally efficient method to start the analysis of nonstationary tidal currents. SSA was first devised by J. M. Colebrook, a British oceanographer, who in 1978 introduced the method to determine the interannual variability of zooplankton abundance in the northeast Atlantic (Colebrook 1978). Eight years later the method was independently developed by mathematicians Broomhead and King (1986), who studied the properties of the method of delays to extract dynamics from experimental data. From this point on, an explosive interest in SSA related to the 
study of chaotic dynamics by mathematicians and physicists [see, e.g., Gibson et al. (1992) and Ghil et al. (2002) and references therein] caused an ever-increasing number of publications in many other areas of science and technology.

It is curious that this method, first introduced in oceanography, has not yet been applied to the study of high-frequency ocean currents to date, although we have used it before in a study of upper-layer lowfrequency currents in the western equatorial Atlantic obtained from multisatellite altimetry (Vianna and Menezes 2003).

The present work explores this new avenue and presents a method of empirical analysis for noisy and nonstationary time series of tidal currents, which is also efficient for short time series. It is very general, in the sense that it is applicable to analysis of currents influenced by many complex processes, as is the case of baroclinic contexts, where the best example is the case of internal tides. It has the advantage of being easy to implement in any computational environment, as we have experienced with our own versions in JAVA and MATLAB.

The method was applied to our ADCP data, which constitutes a simpler barotropic example, where it was able to automatically unravel the observed discrepancies obtained from the preliminary studies using standard methods (e.g., see Table 1).

The work is organized as follows. In section 2 we present a brief historical review of the methods of analysis of tidal phenomena, commenting on some advantages and disadvantages of each, in the context of our work. In section 3 the SSA methodology is described and applied to a synthetic vector current time series that features some of the phenomena of the ADCP data, and the results are compared with those obtained by application of HA and short-term HA (STHA). In section 4 the method is applied to the analysis of the ADCP 30-m-depth mooring data, also comparing SSA to HA and STHA analysis. Section 5 summarizes the main conclusions and future prospects.

\section{Tidal analysis: A short review}

The classical method of local tidal analysis, harmonic analysis, has been maturing for decades since the pioneering work of Sir William Thomson (Lord Kelvin) and George Darwin in the 1870s. Its detailed history is told in the remarkable book by David Cartwright (Cartwright 2000), while the mathematical procedure is well described in many texts (see, e.g., Godin 1972; Pugh 1987). It consists of fitting the observed time series to a finite sinusoidal expansion based on astro- nomically determined and well-known frequencies by least squares. The sinusoidal expansion thus obtained can then be extended to future times, and this constitutes the basis of the classical tidal prediction method. Efficient computer codes for HA soon became available to the international community, as the ones of Foreman (1978) and Franco (1981). Modern computational procedures for HA, based on the Foreman code, are the SLPR2 from the University of Hawaii Sea Level Center (Caldwell 1998) and the most recent MATLAB T_TIDE Toolbox (Pawlowicz et al. 2002).

Notwithstanding the effectiveness of HA for barotropic tidal analysis, offering one of the most successful prediction methods in the whole history of geophysics, its limitations are well known (more details available in Cartwright 2000).

1) The method is unsuitable for nonstationary tidal analysis of noisy data.

2) In HA, only pure spectral lines are considered, and the broadening of these by the continuous spectral background is neglected.

3) Some nontidal oscillations may be interpreted as a superposition of known minor constituents, which are in this case spurious.

Some of these problems were addressed for the first time by the seminal work of Munk and Cartwright (1966) with the introduction of the response function method of analysis. They used the notion that a measured local tidal record is the local response function to the tidal potential forcing, giving more attention to the tidal power spectra, allowing for noise in the measurements. This means bringing the auto- and crosscovariance structures to the center stage in the analysis. The most important finding in their work was that the tidal spectrum was not a simple line spectrum but displayed cusp-shaped spectral peaks, which represented a broadening of the lines by a low-frequency-noise background. Subsequent work (Cartwright 1968) suggested that the noise level found in the tidal band is much higher than that supplied by interactions between tidal and low-frequency variability. It showed that the marked annual modulation of the M2 line was usually higher than the one expected from low-frequency fluctuations in sea level. However, the use of this elegant method has been in part hindered by the complexity of the computations.

One way to circumvent the difficulties related to extraction of the modulations in the tidal species in HA was to use a sliding window to perform what has been called short-term harmonic analysis (Jay and Flinchem 1999). This has the capability to demodulate small perturbing low-frequency coherent signals from the ones in 
the tidal band. However, the latter authors state some of the deficiencies of this boxcar method, which needs short-time windows, and suggest that a CWT-based analysis is more efficient than STHA. One of the important comments relates to the fact that STHA may fail if frequencies contained in the data, not included in the HA frequency (constituent) table, interact with the latter.

Recently, Jay and Kukulka (2003) presented a review of methods of analysis of tidal records containing multiscale nontidal modulations. These authors, among other things, comment on the classical method of complex demodulation (Bloomfield 1976), which can be applied within the framework of STHA or CWT; CD is used with running windows, and uses some filtering (tapering, dependent on some window carpentry) that loses endpoints in the series. Also, oscillations of longer periods than the chosen running window length are not well quantified, and nonstationarity of smaller period components gets masked (Jay and Kukulka 2003).

It should be mentioned here that HA, STHA, CD, and CWT all have in common the fact that they do not use any information of the covariance structure of the data, the importance of which was clearly shown in Munk and Cartwright (1966). In the case of CWT, it seems that it constitutes at most an improvement of Fourier-type analysis applicable to study highly nonstationary data. The CWT method also has a disadvantage of needing to use a filter bank of wavelets and borrows the old classical filtering methods to prevent sideband leakages.

\section{Multiscale current analysis by SSA}

The SSA expansion has the advantage of being capable of decomposing the nonstationary signal into a sum of also nonstationary component reconstructed modes (RCs) ordered in a decreasing sequence of variances (energy) explained by each one, in an automatic way. The number of frequency spectral energy peaks contained in each RC is dependent, however, on a chosen free windowing parameter $M$. They constitute a series of $M$-dependent adaptively filtered versions of the original series, based directly on the data covariance structure, including noise, and are constructed in such a way that partial sums of these RCs increasingly converge into this original series.

The SSA expansion used here is made in the standard way. As explained in Ghil et al. (2002), the starting point of SSA is to embed a time series of $N t$ points $X_{t}$ in a vector space of dimension $M \leq N$ to get a sequence of $M$-dimensional vectors $\left(X_{t}, X_{t+1}, \ldots, X_{t+M-1}\right)$ representing overlapping views of the time series obtained from a sliding $M$-point window. The principal directions in this augmented space of $M$ lagged copies of the time series data is obtained through the KarhunenLoève expansion theorem, based on the lag covariance matrix for the process $X_{t}(1 \leq t \leq N)$, where $X$ is assumed to have zero expectation.

If $X$ is the east $(u)$ or the north $(v)$ current component series of $N$ time points, we may choose an adequate window length $M$ and compute the lag covariance $M \times M$ matrix given by

$$
c_{i j}=\frac{1}{N-|i-j|} \sum_{t=1}^{N-|i-j|} X_{t} X_{t+|i-j|} \quad 0 \leq i, j \leq M-1 .
$$

Eigenvalues $\lambda_{k}$ and eigenvectors $E_{j}^{k}$ of this Toeplitztype matrix are obtained and sorted in descending order of energy $\lambda_{k}$, with $j, k$ from 1 to $M$. The $k$ principal components $p^{k}$ (PCs) are obtained from

$$
p_{i}^{k}=\sum_{j=1}^{M} X_{i+j} E_{j}^{k} \quad 0 \leq i \leq N-M
$$

and the RCs $R^{k}$ by

$$
\begin{array}{rlrl}
R_{i}^{k} & =\frac{1}{M} \sum_{j=1}^{M} p_{i-j}^{k} E_{j}^{k} & M \leq i \leq N-M+1 \\
R_{i}^{k}=\frac{1}{i} \sum_{j=1}^{i} p_{i-j}^{k} E_{j}^{k} & 1 \leq i \leq M-1 \\
R_{i}^{k}=\frac{1}{N-i+1} \sum_{j=i-N+M}^{M} p_{i-j}^{k} E_{j}^{k} & N-M+2 \leq i \leq N .
\end{array}
$$

The windowing parameter $M$ is chosen so that each $R^{k}$ contains a single dominant frequency spectral peak, and a few others with negligible amplitude at most. For the determination of the spectral content of each $R^{k}$ time series, one is free to choose any efficient tool according to personal taste. Although probably the most versatile method would be one using Hilbert transforms to obtain a time-dependent frequency localization, in the lines of Huang et al. (1998), we chose here to use a maximum entropy method (Ghil et al. 2002, and references therein) to get the simplest spectral classification of each RC.

It should be mentioned that the smaller the $M$, the larger the number of these smaller peaks, with more nonstationarity included in the resultant RCs. To completely isolate a low-frequency modulation causing a splitting in a spectral high-frequency peak into RCs with only one spectral component one must choose large $M \mathrm{~s}$ (practice suggests a maximum of $M \leq N / 3$ ). 
The advantage resides in the fact that whatever the choice of $M$, the information content in the $M$ sequence of RCs is the same, and the difference between each choice is only in the way the bookkeeping of the information is done. For smaller $M \mathrm{~s}$ the convergence is also much faster (more variance explained with a smaller number of RCs). This independence is known and has been studied before by Gibson et al. (1992). The observation that each $\mathrm{RC}$ is characterized by a maximum spectral energy peak suggested that indexing each $R^{k}$ with it would enable fast computations for isolation of specific band-limited studies of the signals. Occurrence of zero frequency or periods of the order of the series length represent trends, which may be linear or nonlinear.

In the analysis of one of the scalar components of the vector current, some RCs form a set corresponding visibly to the tidal band, while another set corresponds to large period subtidal signals. One can find several RCs corresponding to the same maximum spectral peak (labeled here as $J$ ), but always each RC corresponds to different variances (eigenvalues of the covariance matrix). The sum of the RCs corresponding to the $J$ peak will give the $J$ waveform (possibly nonstationary), which is the best filtered version of the original series for this specific peak.

To obtain the ellipse parameters from the (possibly modulated) constituent $\left(u_{J}, v_{J}\right)$ series, an EOF analysis is made for the pair of $J$ waveforms, and next a boxcar window of one period of length is applied. Since we intend to use the geographic reference system for the angle of inclination $\theta$ of the major axis relative to the $N$ direction, we compute

$$
\mathrm{EOF}\left(-v_{J}, u_{J}\right)=F^{(J)} ; \quad \operatorname{PC}\left(-v_{J}, u_{J}\right)=B_{(J)},
$$

where $F^{(J)}$ is the rotation $2 \times 2$ matrix, and $B_{(J)}$ is the position vector of evolving ellipses of the $J$ constituent with their axes along the Cartesian axes. The major $a$ and minor $b$ axes may be obtained by the maximum amplitudes of the components of $B_{(J)}$. The angle $\theta_{J}$ may be obtained from

$$
\theta_{J}=\arctan \left(-F_{21}^{(J)} / F_{11}^{(J)}\right),
$$

where care must be taken if $\theta_{J}$ is negative, in which case one must sum it to $2 \pi$ to get the correct angle in the geographic N-S, E-W reference system.

To keep track of the sense of rotation, one may use a signed minor axis $b_{J}$. This sign is obtained from the sign of the vector product of the position vector with the tangent vector of any point of the ellipse, which can be determined at any time index $k$ inside the period in question. Omitting the index $J$ for brevity,

$$
b=\operatorname{sign}\left[B_{2}(k+1) B_{1}(k)-B_{1}(k+1) B_{2}(k)\right] \| .
$$

With this general procedure, any modulations in the ellipse parameters become visible, as shown below, and the sign of $b$ gives the anticyclonic (+) or cyclonic (-) polarization. The time series of the ellipse parameters are not obtainable with HA, which only recovers the average constituents.

One should note that this analysis procedure also can be used for bandpass filtering, with advantages over classical filtering methods. One example where this T_EOF analysis has advantages over standard highpass filtering is the conservation of the structure of the original time series in the filtered one (one does not lose endpoints). In the latter method one does lose a few of the endpoints. The decompositions of the signal into series of different frequency bands, obtained from the PC method, guarantees that by summing these components one recovers the complete original signal. The additional advantage is the automatic estimate of the variance contained in each of these bands.

In the case of vertical current profiles, we first perform a standard EOF analysis to decompose the original signal into sums of products of space- (the EOF) and time- (PC) dependent functions, as usual. Analysis of the PC time components is then done in a similar way by the above-described EOF-based methods in the time domain (T-EOF), the SSA.

\section{Application to a synthetic current signal}

A simple test of this methodology is presented here with a synthetic current signal, its results being compared to those obtained from classical HA analysis and the neoclassical STHA method.

The synthetic current signal is given in Eq. (7), containing an anisotropic modulation of the M2 and S2 tidal ellipse constituents. This kind of modulation mimics a nonlinear anisotropic coupling of a long-period forcing with the tidal band constituents and a linear coupling between the tidal and the subtidal components. Additionally, a long-period signal oriented along $N$ is also present. The M2 and S2 ellipses have their major axes oriented at an angle of $\theta$ relative to $N$. No noise background is considered in this example:

$$
\begin{aligned}
u & =u_{1} \sin \theta-v_{1} \cos \theta, \\
v & =u_{1} \cos \left(\theta+\omega_{a} t\right)+v_{1} \sin \left(\theta+\omega_{a} t\right)+B_{\mathrm{LP}} \sin \left(\omega_{\mathrm{LP}} t\right),
\end{aligned}
$$

where

$$
\begin{aligned}
& u_{1}=\sum_{J} A_{J} \cos \left(\omega_{J} t\right), \\
& v_{1}=\sum_{J} B_{J} \sin \left(\omega_{J} t\right),
\end{aligned}
$$


a

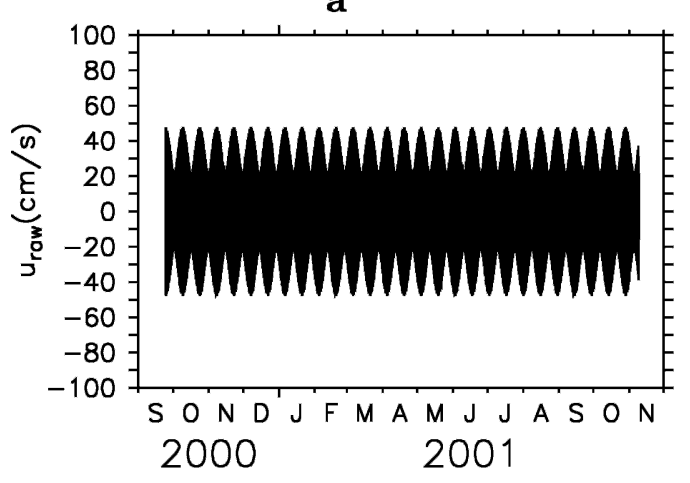

c
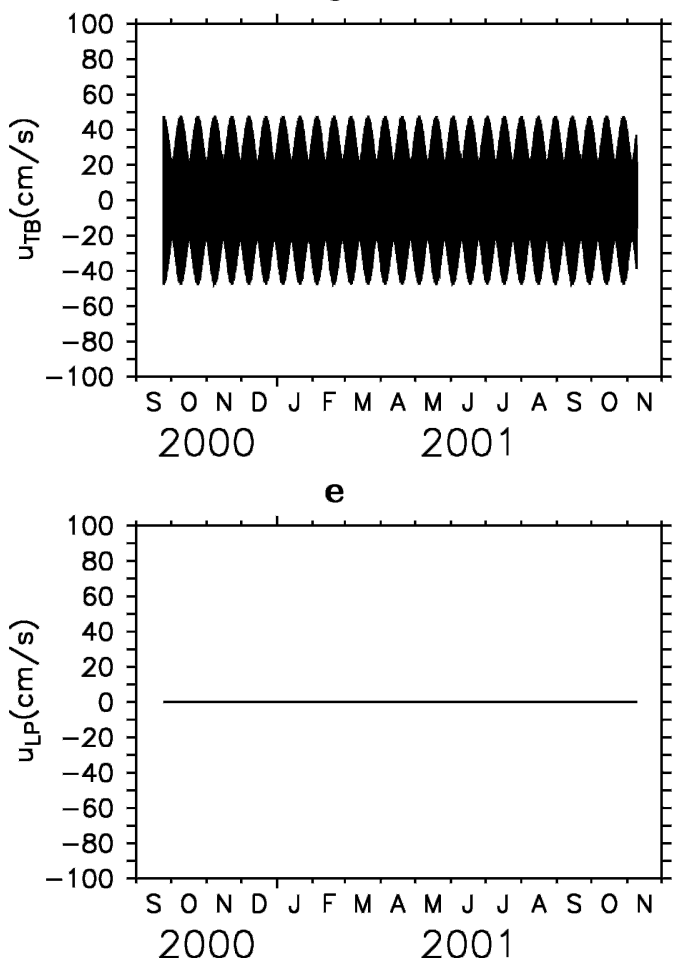

b

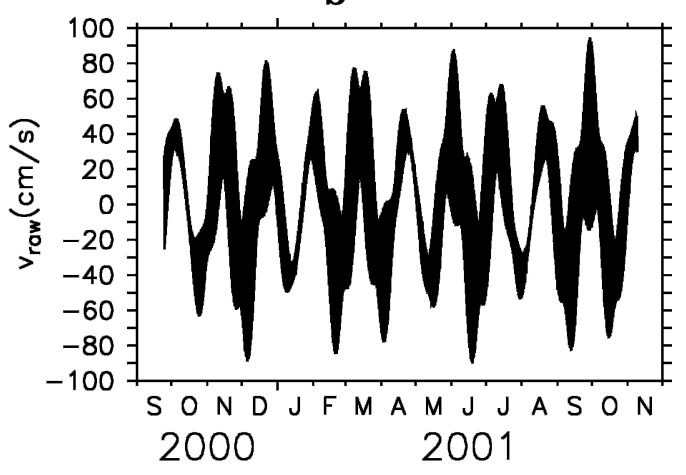

d
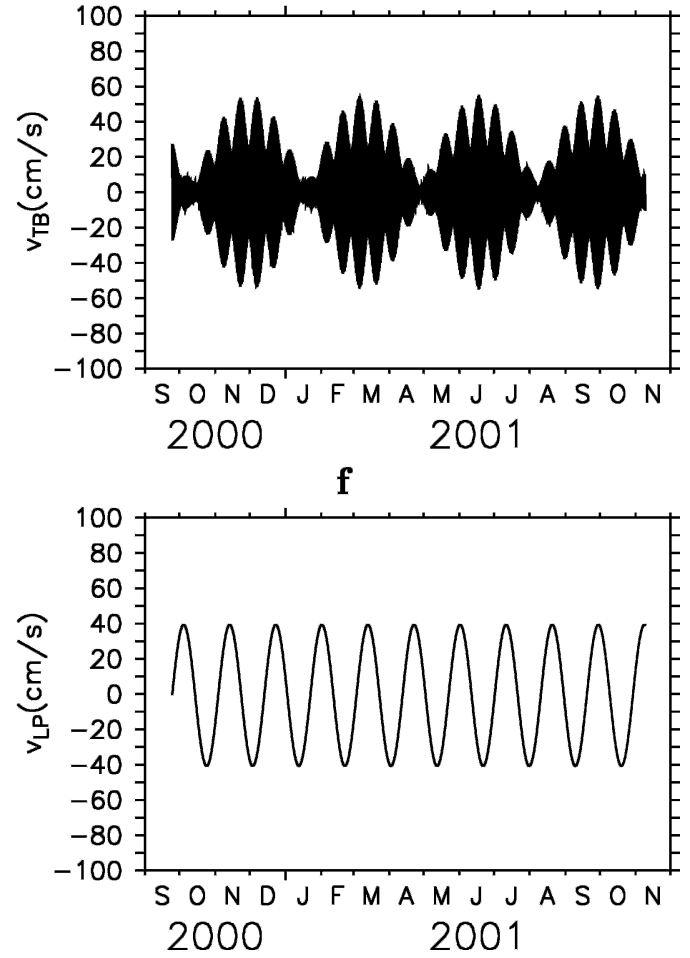

FIG. 1. SSA separation of the synthetic signal into two bands, with $u$ (left) and $v$ (right). (a), (b) Raw data; (c), (d) tidal band; (e), (f) long-period band. Notice that the imposed modulation of 200 days is recovered as part of the tidal band signal (d), and the long-period band $v$ signal of 40 days is recovered as shown in (f).

with $\omega_{J}=2 \pi / T_{J}$, where $T_{J}$ are the periods, and $J=m 2$, $s 2$, refer to the two main semidiurnal tides and the $a$ subscript to the long-period modulation. The subscript LP refers to a long-period signal. The numerical values for the amplitudes are $A_{m 2}=40, A_{s 2}=15, B_{m 2}=5, B_{s 2}$ $=2, B_{\mathrm{LP}}=40, T_{a}=200, T_{\mathrm{LP}}=40$ (periods in days), while the semidiurnal periods are 12.42 and $12.00 \mathrm{~h}$, and $\theta=60^{\circ}$. The time step is defined to be $1 \mathrm{~h}$, and the length is 410 days.

Although the SSA analysis is equally efficient with or without previous high-pass filtering to analyze the tidal band, the classical and neoclassical methods are not. A straightforward analysis of the synthetic vector signal with the use of T_TIDE gave a percent predicted/total variance of 42.8, while analysis of the high-passed signal gave 72.8. Therefore, we separated the signal by use of SSA into a tidal band $(2.5 \mathrm{~h}<T \leq 26 \mathrm{~h})$ and a lowfrequency band $(T>26 \mathrm{~h})$ for ease of comparison.

Figure 1 shows the input signal and separated bands, where the value of the windowing parameter was chosen arbitrarily to be $90 \mathrm{~h}$. This band separation, which is accomplished by summing RCs, is independent of $M$, because this parameter only controls the number and form of the component RCs necessary to obtain the 
TABLE 2. Results of HA with the synthetic current data using T_TIDE, exhibiting the significant constituents with major axes larger than $0.01 \mathrm{~cm} \mathrm{~s}^{-1}$. Inclination angle relative to east, measured counterclockwise.

\begin{tabular}{lcccrr}
\hline \hline Constituents & Frequency & $a\left(\mathrm{~cm} \mathrm{~s}^{-1}\right)$ & $b\left(\mathrm{~cm} \mathrm{~s}^{-1}\right)$ & Inclination $\left(^{\circ}\right)$ & Phase \\
\hline SSA & 0.0002282 & $0.013 \pm 0.004$ & $-0.002 \pm 0.00$ & $90.31 \pm 7.53$ & $313.40 \pm 17.76$ \\
MSF & 0.0028219 & $0.010 \pm 0.004$ & $0.003 \pm 0.00$ & $95.57 \pm 14.31$ & $348.79 \pm 24.52$ \\
ALP1 & 0.0343966 & $0.015 \pm 0.005$ & $-0.003 \pm 0.00$ & $95.85 \pm 9.51$ & $26.70 \pm 18.99$ \\
2Q1 & 0.0357064 & $0.011 \pm 0.005$ & $-0.004 \pm 0.00$ & $103.98 \pm 17.37$ & $242.59 \pm 26.50$ \\
SIG1 & 0.0359087 & $0.011 \pm 0.005$ & $-0.004 \pm 0.00$ & $104.72 \pm 14.87$ & $22.03 \pm 28.42$ \\
TAU1 & 0.0389588 & $0.016 \pm 0.005$ & $-0.003 \pm 0.00$ & $95.54 \pm 9.35$ & $145.80 \pm 19.71$ \\
BET1 & 0.0400404 & $0.010 \pm 0.005$ & $0.004 \pm 0.00$ & $94.11 \pm 18.50$ & $181.05 \pm 35.04$ \\
NO1 & 0.0402686 & $0.012 \pm 0.005$ & $-0.004 \pm 0.00$ & $103.11 \pm 17.20$ & $2.63 \pm 27.35$ \\
CHI1 & 0.0404710 & $0.011 \pm 0.005$ & $-0.004 \pm 0.00$ & $106.27 \pm 15.73$ & $138.70 \pm 26.28$ \\
PI1 & 0.0414385 & $0.020 \pm 0.005$ & $0.002 \pm 0.00$ & $88.55 \pm 6.23$ & $65.58 \pm 13.77$ \\
P1 & 0.0415526 & $0.014 \pm 0.005$ & $0.003 \pm 0.00$ & $88.92 \pm 10.90$ & $356.67 \pm 21.93$ \\
PSI1 & 0.0418948 & $0.014 \pm 0.005$ & $-0.003 \pm 0.00$ & $95.28 \pm 9.08$ & $306.79 \pm 20.93$ \\
PHI1 & 0.0420089 & $0.021 \pm 0.005$ & $-0.002 \pm 0.00$ & $90.99 \pm 6.27$ & $84.46 \pm 14.22$ \\
SO1 & 0.0446027 & $0.010 \pm 0.005$ & $0.005 \pm 0.00$ & $97.20 \pm 24.07$ & $124.65 \pm 38.33$ \\
001 & 0.0448308 & $0.014 \pm 0.005$ & $-0.004 \pm 0.00$ & $100.34 \pm 11.48$ & $305.34 \pm 26.87$ \\
M2 & 0.0805114 & $34.689 \pm 0.113$ & $0.816 \pm 3.36$ & $179.62 \pm 5.81$ & $350.34 \pm 0.24$ \\
MKS2 & 0.0807396 & $16.199 \pm 3.814$ & $0.329 \pm 0.08$ & $90.60 \pm 0.36$ & $160.21 \pm 12.23$ \\
S2 & 0.0833333 & $12.998 \pm 0.172$ & $0.435 \pm 3.29$ & $179.31 \pm 15.26$ & $265.68 \pm 1.10$ \\
K2 & 0.0835615 & $5.928 \pm 3.703$ & $0.016 \pm 0.08$ & $90.35 \pm 0.69$ & $76.49 \pm 36.11$ \\
\hline
\end{tabular}

filtered signals. Anyhow, we tested the use of several different $M$ s, starting with $13 \mathrm{~h}$, up to 1440 (60 days), obtaining the same results. The tidal band explained $100 \%$ of the variance for $u$, and $36 \%$ for $v$, and the long period gave $0 \%$ for $u$ and $64 \%$ for $v$. The 40-day signal is therefore immediately recovered from the longperiod band (since only one sinusoid was imposed) for $v$.

The analysis of the tidal period band was done using $M=480$, that is, 20 days, chosen to mimic the usual minimum hourly record length used in classical hydrographic work. It should be stated that in this particular case the choice of $M$ does not affect the final results, and does not even affect the individuals RCs.

The analysis returned only two ellipse constituents: M2 and S2. For each vector component, one finds the first two RCs corresponding to M2 (the cosine and the sin-type terms necessary to adjust the correct phase) and, analogously, the two subsequent ones to S2. The other higher-order RCs correspond to a negligible energy plateau and were discarded, although they had the same M2 and S2 frequencies. The total variances for M2 and S2 were $88.37 \%$ and $11.51 \%$, respectively, for $u$ (totalling $99.88 \%$ ) and $88.46 \%$ and $11.47 \%$ for $v$ (totalling $99.93 \%$ ).

The results were compared with those obtained from HA and STHA. For STHA we used HA recursively with a window of the same $480 \mathrm{~h}$, and a time step of 1 $\mathrm{h}$, to better compare with the SSA. In this latter case the computation of the time series of ellipse parameters was made using a 1-h time step for a 13-h running window, representing approximately the period of the semidiurnal species.
Table 2 presents the results for HA, where only the significant constituents with amplitudes exceeding 0.01 $\mathrm{cm} \mathrm{s}^{-1}$ are included for brevity. One noticeable problem with $\mathrm{HA}$ is the fact that although the recovered $u$ variance is $99.6 \%$, the recovered modulated component $v$ variance is only $32.4 \%$. This means that the modulation is the source of the high-amplitude noise, since the imposed $T_{a}=200$ day modulating period was not detected. Instead, a series of small (but found to be significant) periods that were not part of the simulated signal is obtained.

Figure 2 illustrates the time series of the major/minor axes, and major axis inclination angle for M2 in the geographic frame of reference, obtained with the SSA and the STHA. The smaller-amplitude $\mathrm{S} 2$ component is just parallel to the M2 but was omitted here. It should be noted that if we use a 13 -h running window to get the M2 ellipse parameter evolution with SSA, we lose 12 endpoints in the process, while for STHA we lose a total of 480 endpoints. The minor axis $b$ has negative values in some time intervals, and these represent, as usual, a change in the sense of rotation. These effects would not appear with an imposed isotropic modulation. The periodicity of the $b$ s and $\theta$ s is 200 days, while that of $a$ is about 100 days.

As can be observed in Fig. 2, the analysis with STHA gives essentially the same results as SSA for this example, but the computation with STHA is slower by a factor of 150 relative to the SSA method. The variances recovered by each running STHA analysis window varied between $30 \%$ and $99 \%$ along the sample series and still finds spurious constituents, which suggests that this 

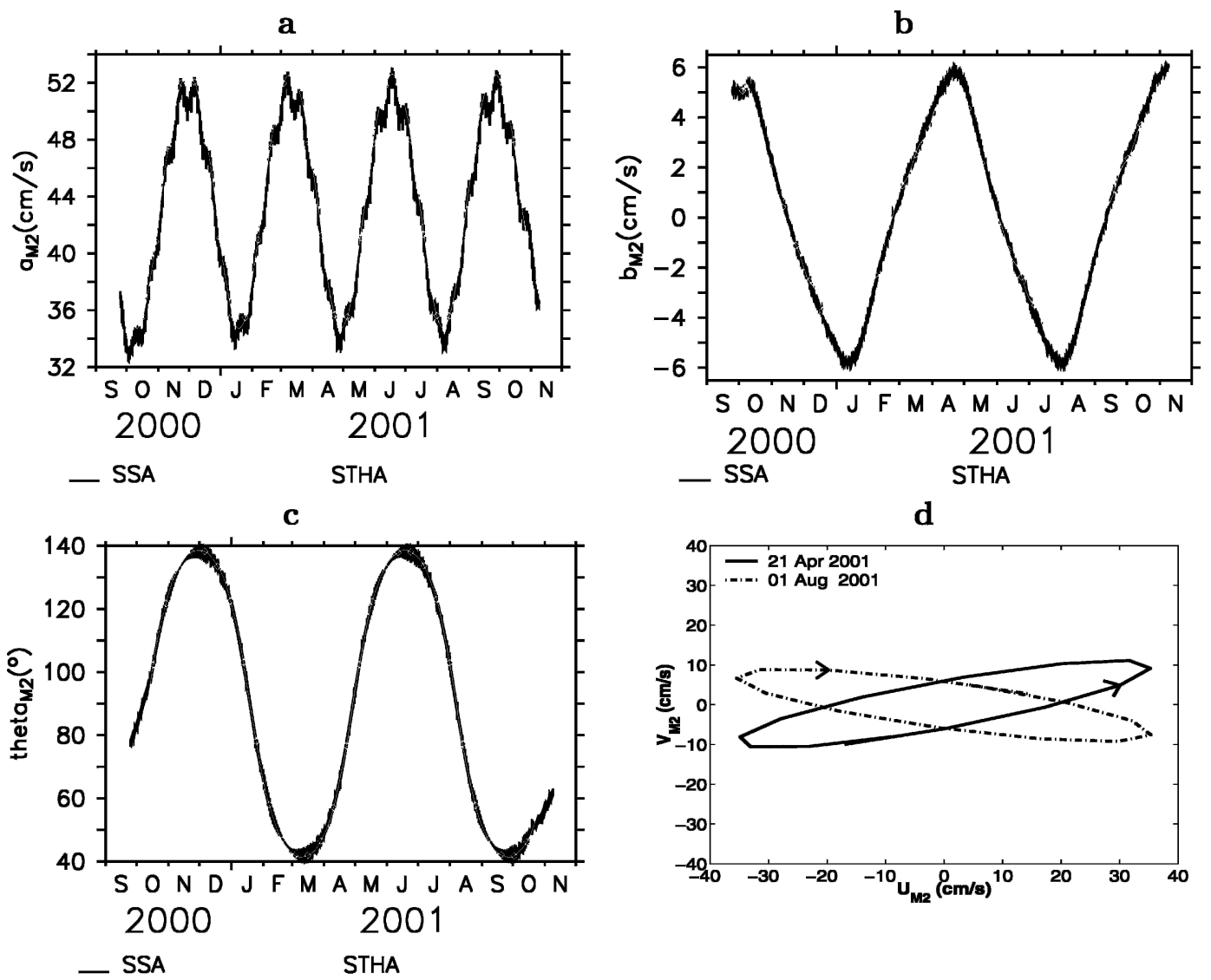

FIG. 2. The M2 ellipse parameter time series for the synthetic signal, computed with SSA (black line) and STHA (gray line), which is 150 times slower than SSA. (a) Major axis; (b) minor axis; (c) clockwise inclination angle relative to true north $\mathrm{N}$; (d) M2 ellipses at one maximum of $b$ (anticyclonic rotation) and one minimum of $b$ (cyclonic rotation), computed with SSA only.

analysis method might in addition be not robust and is unstable.

\section{Application to ADCP data \\ a. Data acquisition}

An RDI 300-kHz Workhorse Sentinel ADCP with a CPU Firmware 8.2, and equipped with bottom pressure and temperature sensors, was positioned at a distance of $42 \mathrm{~km}$ from the coast, near the shelf break, at 30-m depth, as shown in Fig. 3.

The exact site was chosen considering, among other things, the interest in minimizing the cost/benefit ratio for the complete deployment/retrieval work. This required a preliminary survey of the area through imageassisted mapping of the seafloor, using Landsat Thematic Mapper (TM) satellite data and diving, in the same way as reported by Vianna et al. (1991, 1993), Santos (1999), and Testa and Bosence (1999). The area was seen to be dominated by sand dune fields (less than 5-m heights and about 1-km span length) and an almost linear beach rock ridge of maximum height of $5 \mathrm{~m}$ (known as a Risca), with the dunes and the ridge having their crests parallel to the bathymetric gradients. The site was windward from the ridge, at an estimated distance of $60 \mathrm{~m}$ from it. The diving platforms were local lobster fishing boats equipped with GPS, easily available to us at any time.

The ADCP was programmed to obtain current velocity profiles, operating with bursts of 240 pings, a veraged in $2 \mathrm{~min}$ at each half hour. The depth cell size was $0.5 \mathrm{~m}$, giving a total of 50 useful bins for this 27-30-m-depth site (around $3 \mathrm{~m}$ of tidal amplitude). The first bin was at $2.76 \mathrm{~m}$ and the last one at $27.26 \mathrm{~m}$ from the seafloor. The sound speed computation used the temperature and pressure sensor data and an assumed salinity of 36 psu. The expected velocity accuracy of this scheme was $1.8 \mathrm{~cm} \mathrm{~s}^{-1}$. See Table 3 for details.

The fieldwork was done between September 2000 and November 2001, with three continuous data segments retrieved at the dates shown in Table 4. Notice 


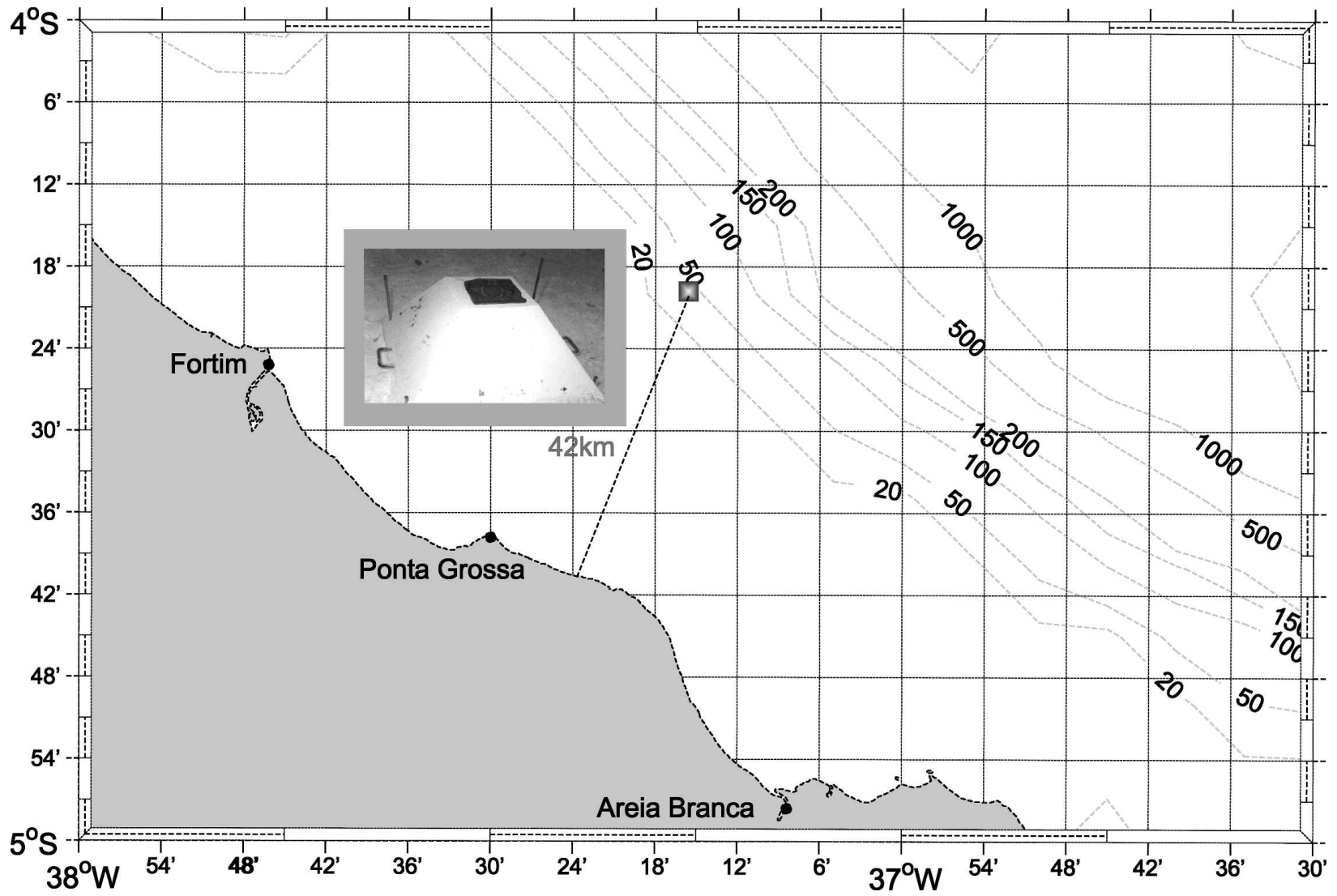

FIG. 3. Geographic setting of the ADCP mooring at $4^{\circ} 19^{\prime} 34^{\prime \prime} \mathrm{S}, 37^{\circ} 15^{\prime} 36^{\prime \prime} \mathrm{W}$, with the bathymetric contours in $\mathrm{m}$. The inset shows the trawl-proof pyramid made of nonmagnetic materials on site.

that 2-day data gaps between segments resulted from the operations of instrument retrieval and redeployment at the same fixed mooring pyramid.

\section{b. Preprocessing}

The data segments were first subsampled to form hourly data time series and then rotated from the original magnetic $\left(21^{\circ} \mathrm{W}\right)$ to the geographic reference axes.

The 2-day gaps were filled with an interpolation pro-

TABLE 3. ADCP parameter settings.

\begin{tabular}{ll}
\hline \hline \multicolumn{1}{c}{ Parameter } & \multicolumn{1}{c}{ Choice } \\
\hline Ensemble interval & $30 \mathrm{~min}$ \\
Pings per ensemble & 240 \\
Time between pings & $0.5 \mathrm{~s}$ \\
Ping weighting & Triangle \\
Depth cell size & $0.5 \mathrm{~m}$ \\
Number of depth cells & 50 \\
Error velocity threshold & $2.5 \mathrm{~m} \mathrm{~s}^{-1}$ \\
Heading alignment & $0^{\circ}\left(\mathrm{magnetic}^{-1}\right.$ reference axes) \\
Coordinate transformation & Earth coordinates \\
Time reference & UTC \\
\hline
\end{tabular}

cedure that consisted of separating the signal at each bin into the tidal and long-period bands by SSA. The gap in the long-period band was filled using cubic spline interpolation, while for the tidal band the HA prediction method was used. Finally, by summing these signal components, we are able to restore the total 410-day dataset, but now without any gap. The simpler direct use of the HA with T_TIDE without taking into account the long-period band causes a jump difference on the filled gap.

\section{c. Vertical EOF expansion}

To represent the data in a more compact form, vertical EOF expansions were made independently for the $u$ and $v$ profiles (with the mean profiles removed but kept for further use). We did not use complex EOFs because we know that one component might contain modulations that are absent in the other, rendering the joint correlation analysis a less efficient method.

The first EOF explained $98 \%$ of the total variance, and all the others are part of the nonconvergent variance plateau (each one explains less that $0.1 \%$ of the 
TABLE 4. Data acquisition timetable exhibiting the initial and final dates for each data segment.

\begin{tabular}{cccr}
\hline \hline Data segment & Start & End & Ensembles \\
\hline 1 & 1500 UTC 24 Sep 2000 & 1500 UTC 2 Dec 2000 & 3312 \\
2 & 1400 UTC 4 Dec 2000 & 1300 UTC 7 Mar 2001 & 4455 \\
3 & 1600 UTC 9 Mar 2001 & 1100 UTC 9 Nov 2001 & 11751 \\
\hline
\end{tabular}

variance), representing white noise. The EOF profile showed that the flow is barotropic, in such a way that the maximum amplitudes occur at midwater, decreasing only very slightly to the surface or to the bottom.

The separation of the respective PC1s into tidal and long-period bands showed that $56 \%$ of the variance in $u$ and $61 \%$ in $v$ is contained in the tidal band, while $42 \%$ in $u$ and $36 \%$ in $v$ is contained in the long-period band. The value of $M$ used in the SSA for this separation was $90 \mathrm{~h}$, adequate to separate well the semidiurnal and diurnal constituents with our method. In Fig. 4 the quality-controlled raw input vertical mean data,
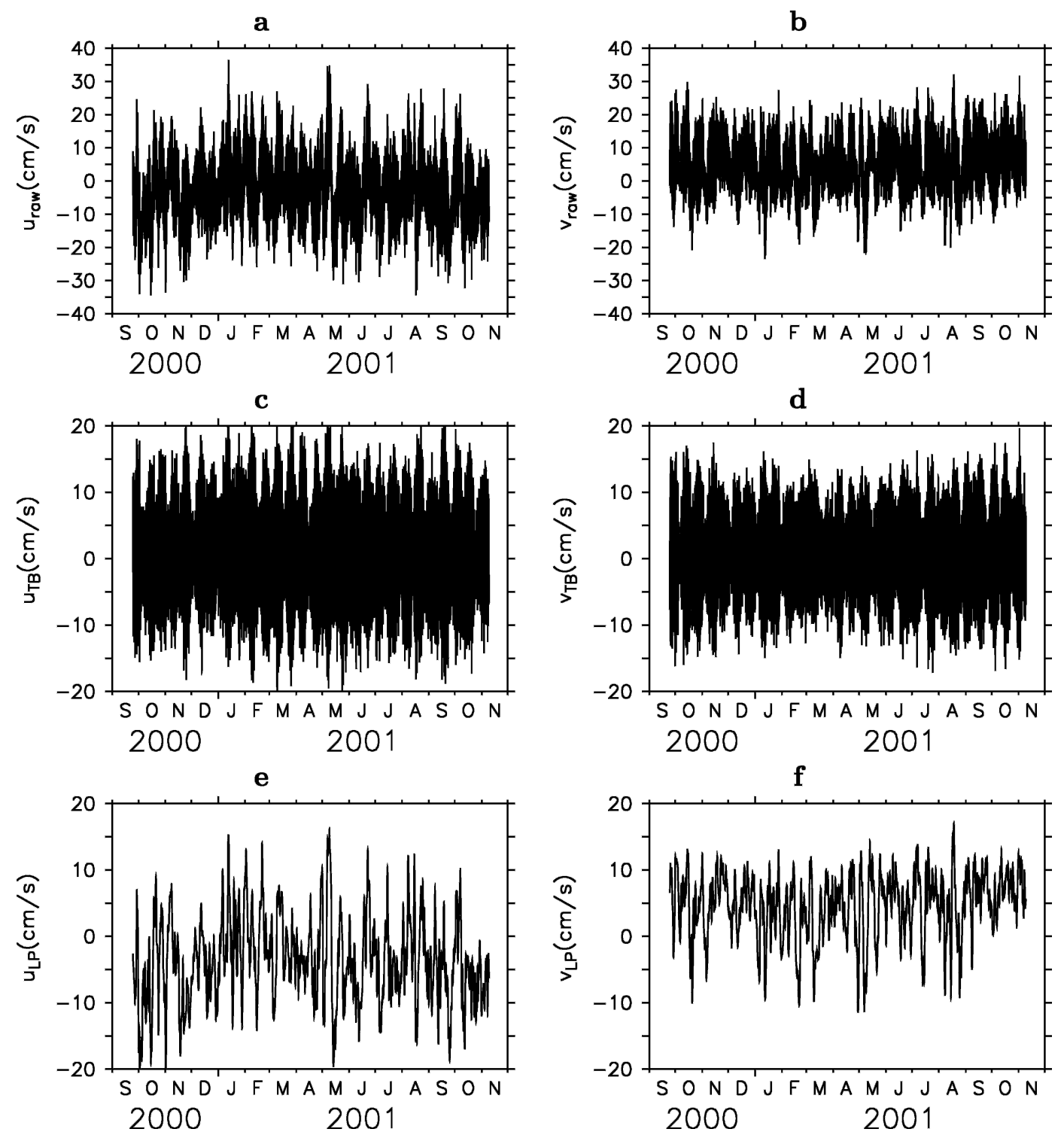

FIG. 4. ADCP signal separation into two bands, with $u$ (left) and $v$ (right). (a), (b) Raw data; (c), (d) tidal band; (e), (f) long-period band. Both the tidal and long-period bands were analyzed, with the results for the tidal band shown in Table 5. 
and the separated vertical mean band signals, are displayed.

The EOF analysis permitted us to filter out the random spikes at different bins, while the band separation allowed the RCs related to energy peaks of less than 2.5 -h period to be discarded. This enabled the construction of a quality-controlled 410-day hourly profile dataset through reprojection of the filtered $u$ and $v$ signals back into the 50-bin space, resulting in the final separated tidal and long-period current profile datasets. In this reprojection, the mean vertical profile was summed to the long-period band signal. Although this exercise may look sterile in our context, it would be of fundamental importance in the case of baroclinic current profiles, as in the case of internal tides, and this is one of the reasons that we provide this detailed description here.

\section{d. Current analysis by SSA}

Although the described SSA analysis procedures do not require a separation of the vector signals into two different bands (tidal and long period), this was done to more easily compare with HA and STHA of the same data.

Analysis of the tidal band with SSA and using $M=$ $480 \mathrm{~h}$ (20 days) gives the main ellipse constituents whose peak periods correspond to the well-known tidal periods. If we use, for example, $M=13 \mathrm{~h}$, the RCs can already see D2 and D4 and, surprisingly, D1. Increasing $M$ to $96 \mathrm{~h}$, the method gets M2, S2, the D1 constituents, and M4. The spectral peaks are usually cusplike and get narrower the larger the $M$. The component RCs, with strong modulations for small $M \mathrm{~s}$, transfer their energy into other less-modulated RCs as $M$ grows, but the information contained in the RC series remains the same.

We also tested what is the minimum number of points in the time series one needs to use for tidal analysis with SSA. With $25 \mathrm{~h}$ of data one is able to identify the D2 and D4 species; with $49 \mathrm{~h}$ we are able to see D4 and the splitting of D2 (into M2 and S2), and detect the D1 species; with $97 \mathrm{~h}$ we get M4 and the major semidiurnal constituents, plus the diurnals. Except for the 25-h case, where the HA with T_TIDE returns a Rayleigh criterion violation between $\mathrm{K} 1$ and M2, the two methods give consistent results, but HA gives out many spurious constituents, while SSA does not. We conclude that SSA does not have any major limitation with short datasets, in contrast to CWT [Jay and Kukulka (2003) state that CWT needs at least 4 days of data to be effective].

Table 5 exhibits the dominant empirical tidal species found, corresponding to the first $10 \mathrm{RCs}$ together with the respective variances explained for each of $u$ and $v$.
TABLE 5. Results of analysis of the ADCP tidal band signal with detected periods of the tidal constituents and respective percent variances for $u$ and $v$. The corresponding tabulated tidal periods are included.

\begin{tabular}{ccccc}
\hline \hline Constituents & $T_{\text {tab }}(\mathrm{h})$ & $T_{\text {obs }}(\mathrm{h})$ & $\begin{array}{c}\text { Percent } \\
\text { variance }(u)\end{array}$ & $\begin{array}{c}\text { Percent } \\
\text { variance }(v)\end{array}$ \\
\hline M2 & 12.42 & 12.41 & 66.47 & 74.07 \\
S2 & 12.00 & 12.01 & 10.01 & 9.45 \\
N2 & 12.66 & 12.68 & 4.25 & 2.79 \\
K1 & 23.94 & 23.95 & 3.28 & 1.6 \\
M4 & 6.21 & 6.21 & 2.08 & - \\
\hline
\end{tabular}

The tabulated values of the corresponding astronomically determined (as opposed to empirically determined) tidal periods are included for constituent identification of the periods obtained without prior knowledge of the known tabulated values.

Table 6 offers the results obtained from the HA using T_TIDE. For the most significant and highestamplitude constituents, the notable difference is in the D1 species. While SSA finds only K1, HA finds O1, P1, S1, and K1, and while SSA finds long-period modulations, HA does not.

Figure 5 shows the evolution of the M2 ellipse parameters computed with SSA and STHA, clearly exhibiting long-period modulations. Spectral analysis of the inclination angle $\theta_{\mathrm{M} 2}$ gives as main spectral peaks the annual (72\%) and the 57-day periods (20\%). It can be seen that the minor axis changes sign, which indicates a change from anticyclonic (positive $b_{\mathrm{M} 2}$ ) to cyclonic (negative $b_{\mathrm{M} 2}$ ) rotation. The $\mathrm{S} 2$ ellipse parameter variation is analogous and is omitted for brevity. The SSA analysis was also made for the long-period signal, and it gave the same annual and 57-day spectral peaks. This signal was found to be strongly polarized along the bathymetric contours and almost parallel to the minor axis of the tidal ellipses (not shown here).

It should be stressed that the detection of a 57-day period in both the long-period and inclination-angle signals is a natural consequence of the SSA analysis. This result is not obtainable by use of HA or STHA. It can be stated that detection of this spectral component in both variables may be considered as evidence of the existence of some interaction between them. The presence of 50-day oscillations in current meter data from the North Brazil Current has been thoroughly described by Johns et al. (1990) and is here recorded for the first time for the northeast Brazilian shelf south of the equator.

\section{Conclusions}

An alternative method of empirical analysis applicable for nonstationary tidal and nontidal current data 
TABLE 6. Results of HA of the ADCP data using T_TIDE, exhibiting the significant constituents with major axes larger than 0.4 $\mathrm{cm} \mathrm{s}^{-1}$. Inclination angle relative to east, measured counterclockwise.

\begin{tabular}{lccccc}
\hline \hline Constituents & Frequency & $a\left(\mathrm{~cm} \mathrm{~s}^{-1}\right)$ & $b\left(\mathrm{~cm} \mathrm{~s}^{-1}\right)$ & Inclination $\left(^{\circ}\right)$ & Phase \\
\hline O1 & 0.0387307 & $0.830 \pm 0.186$ & $-0.335 \pm 0.19$ & $170.12 \pm 18.22$ & $155.65 \pm 16.64$ \\
P1 & 0.0415526 & $0.718 \pm 0.188$ & $0.046 \pm 0.20$ & $165.29 \pm 15.75$ & $179.06 \pm 16.08$ \\
S1 & 0.0416667 & $1.878 \pm 0.201$ & $0.365 \pm 0.19$ & $149.06 \pm 6.22$ & $114.43 \pm 5.23$ \\
K1 & 0.0417807 & $0.429 \pm 0.204$ & $-0.074 \pm 0.18$ & $6.23 \pm 24.75$ & $359.44 \pm 26.43$ \\
N2 & 0.0789992 & $2.420 \pm 0.323$ & $-0.114 \pm 0.35$ & $32.50 \pm 8.09$ & $288.93 \pm 8.47$ \\
NU2 & 0.0792016 & $0.547 \pm 0.362$ & $-0.047 \pm 0.30$ & $3.13 \pm 38.75$ & $314.48 \pm 35.19$ \\
M2 & 0.0805114 & $11.461 \pm 0.320$ & $1.174 \pm 0.35$ & $41.90 \pm 1.88$ & $302.67 \pm 1.85$ \\
H2 & 0.0806255 & $0.878 \pm 0.355$ & $0.048 \pm 0.38$ & $134.25 \pm 21.63$ & $196.63 \pm 22.71$ \\
L2 & 0.0820236 & $0.558 \pm 0.338$ & $0.051 \pm 0.33$ & $173.43 \pm 35.70$ & $107.93 \pm 38.21$ \\
T2 & 0.0832193 & $0.622 \pm 0.387$ & $0.149 \pm 0.36$ & $147.26 \pm 36.01$ & $51.76 \pm 36.15$ \\
S2 & 0.0833333 & $4.049 \pm 0.366$ & $-0.247 \pm 0.38$ & $38.47 \pm 5.03$ & $322.01 \pm 5.20$ \\
R2 & 0.0834474 & $0.469 \pm 0.329$ & $-0.022 \pm 0.29$ & $142.04 \pm 46.33$ & $32.23 \pm 49.70$ \\
K2 & 0.0835615 & $0.985 \pm 0.349$ & $0.148 \pm 0.40$ & $32.01 \pm 21.89$ & $337.43 \pm 24.43$ \\
MN4 & 0.1595106 & $0.488 \pm 0.163$ & $-0.000 \pm 0.12$ & $21.72 \pm 14.76$ & $243.48 \pm 17.73$ \\
M4 & 0.1610228 & $1.601 \pm 0.149$ & $0.016 \pm 0.12$ & $24.40 \pm 4.51$ & $260.71 \pm 5.16$ \\
MS4 & 0.1638447 & $1.077 \pm 0.158$ & $0.178 \pm 0.14$ & $20.34 \pm 7.30$ & $284.01 \pm 8.37$ \\
\hline
\end{tabular}

a

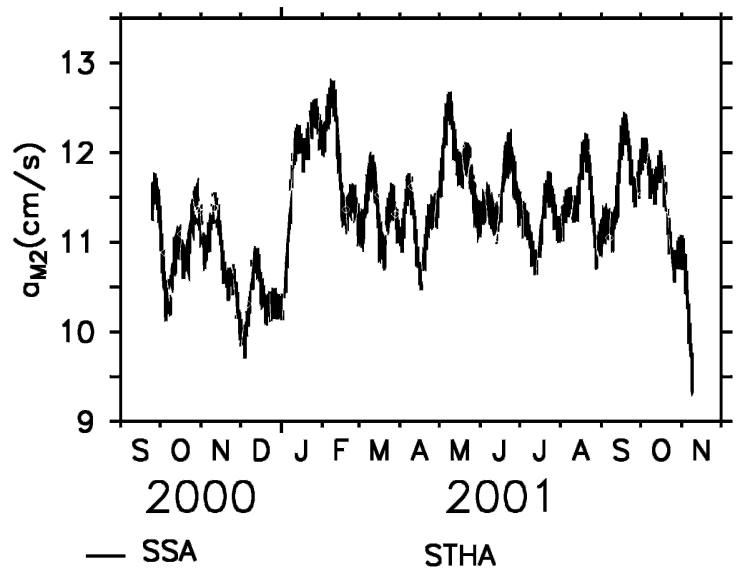

C

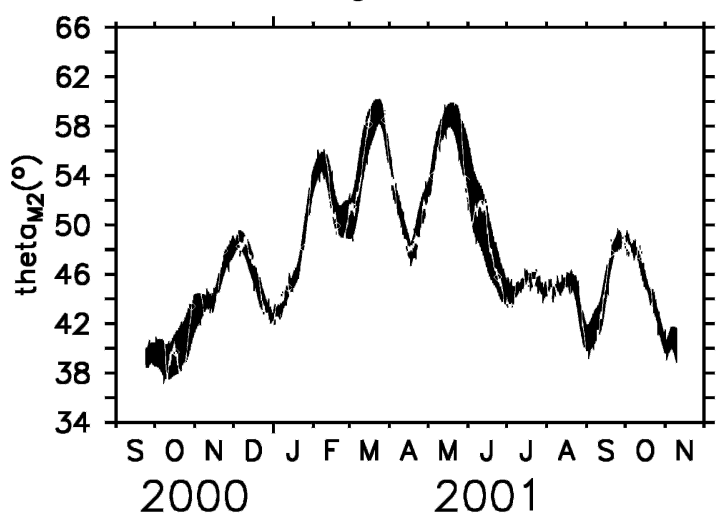

b

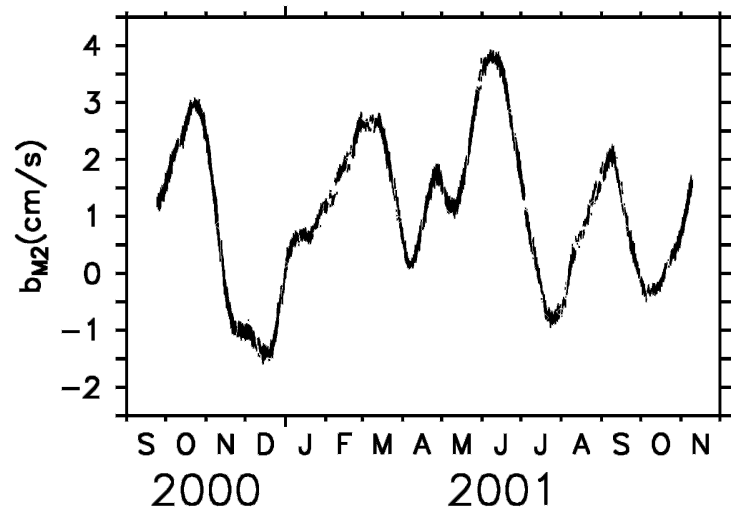

- SSA

STHA

d

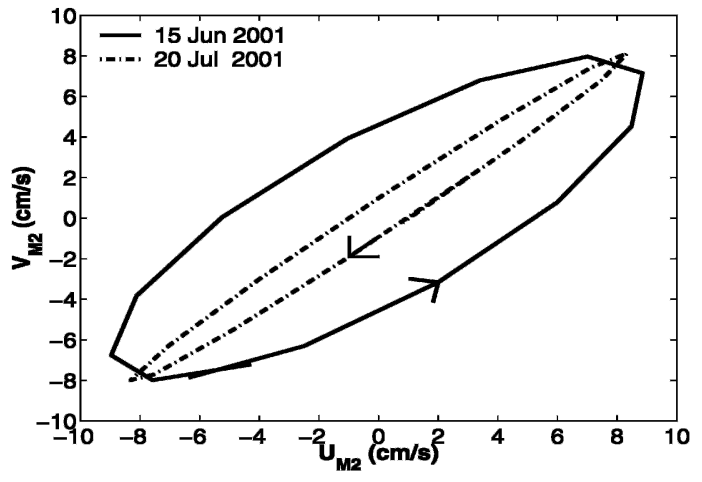

- SSA

STHA

FIG. 5. The M2 ellipse parameter time series for the ADCP signal, computed with SSA (black line) and STHA (gray line), which is 150 times slower than SSA. (a) Major axis; (b) minor axis; (c) clockwise inclination angle relative to true north N; (d) M2 ellipses at one maximum of $b$ (anticyclonic rotation) and one minimum of $b$ (cyclonic rotation). The angle relative to true north $\mathrm{N}$ exhibits dominance by an annual and a 57-day modulation. 
was presented, based on the singular spectrum reconstructed components (SSA-RC) methodology. One important advantage of this methodology resides in the fact that it is centered upon statistics based on the sample lag covariance matrices estimated through the use of a free windowing parameter $M$ that may be adjusted for each application.

We showed that the method is flexible, fast, and very efficient for both short and long time series, and very easy to implement. It separates trends, oscillatory patterns, and noise in a single operation via the reconstructed modes (RCs), which are obtained from the eigenvectors of an extended $M \times M$ lag covariance matrix. The sequence of RCs (themselves nonstationary) represents a sequence of filtered versions of the zero expectation value series obtained from the original one. The choice of $M$ does not change the information content obtained in the analysis but influences how the bookkeeping of this information (through morestationary or less-stationary RCs) is preferred for each particular problem.

As shown long ago by Munk and Cartwright (1966), the use of information from the power spectrum, and therefore from auto and cross covariances, is important to recover the physics in the tidal band and the interaction of tidally forced oscillations with other oceanic and meteorological forcings. Their response-type analysis, if coupled to the present methodology, could possibly be made easier to apply.

The method was applied to a synthetic dataset, and to well-behaved current data from a barotropic setting obtained from the northeast Brazilian shelf. It was compared with HA and STHA, giving consistent results with those obtained with STHA, but because the SSA method also applies to the long-period part of the total signal, it can be considered as superior to HA and STHA. Additionally, the SSA method was 150 times faster than STHA.

We showed that even in the case of well-behaved bathymetric contours, and no river discharge, one is able to get better results than those obtainable from standard analysis, that is, determine important and subtler effects not made apparent by direct application of the harmonic method. However, it might have as its most important application the analysis of internal tides and the complex coupled interactions generally observed in baroclinic contexts.

The subsequent long-term prediction operation, not described here, can be implemented to extend into the future the RCs obtained in the analysis. Also, we may improve the method of spectral analysis of each RC by use of Hilbert transforms, instead of the well-known maximum entropy method (MEM).
Acknowledgments. This methodology was developed by the authors in the last few years as part of an effort made in VM Oceanica, related to research and development of more efficient software for real-time monitoring of ocean currents, and associated data quality control. We are indebted to two anonymous reviewers for constructive criticism, and to Rich Paulowicz and Bob Beardsley for making their T_TIDE code available to the community. We acknowledge also the efforts made by our diving team members Jose (Dede) Bezerra Neto and Viviane Vianna, who assisted us in the deployment/recovery operations.

\section{REFERENCES}

Beardsley, R. C., J. Candela, R. Limeburner, W. R. Geyer, S. J. Lentz, B. M. Castro, D. Cacchione, and N. Carneiro, 1995: The $\mathrm{m} 2$ tide on the Amazon shelf. J. Geophys. Res., 100 (C2), 2283-2319.

Bloomfield, P., 1976: Fourier Analysis of Time Series: An Introduction. John Wiley \& Sons, 258 pp.

Broomhead, D. S., and G. P. King, 1986: Extracting qualitative dynamics from experimental data. Physica, 20D, 217-236.

Caldwell, P., 1998: Sea level data processing on IBM-PC compatible computers. Joint Archive for Sea Level of the National Oceanography Data Center and University Hawaii Sea Level Center, Tech. Rep. JIMAR Contribution 98-319, 80 pp.

Cartwright, D., 1968: An unified analysis of tides and surges round north and east Britain. Philos. Trans. Roy. Soc. London, 1-5.

Cartwright, D. E., 2000: Tides: A Scientific History. Cambridge University Press, 292 pp.

Colebrook, J. M., 1978: Continuous plankton records: Zooplankton and environment, North-East Atlantic and North Sea, 1948-1975. Oceanol. Acta, 1, 9-23.

Foreman, M. G. G., 1978: Manual for tidal currents analysis and predictions. Pacific Marine Science Rep. 78-6, Institute of Ocean Sciences, Patricia Bay, Sidney, BC, Canada, 57 pp.

Franco, A., 1981: Tides: Fundamentals, analysis and prediction. Institute de Pesquisas Tecnologicas do Estado de Sao Paulo, Publ. 1182, 232 pp.

Ghil, M., and Coauthors, 2002: Advanced spectral methods for climatic time series. Rev. Geophys., 40, 1003, doi:10.1029/ 2000RG000092.

Gibson, J. F., D. Farmer, M. Casdagli, and S. Eubank, 1992: An analytic approach to practical state space reconstruction. Physica D, 57, 1-30.

Godin, G., 1972: The Analysis of Tides. University of Toronto Press, 264 pp.

Huang, N. E., and Coauthors, 1998: The empirical mode decomposition and the Hilbert spectrum for non-linear and nonstationary time series analysis. Proc. Roy. Soc London, A454, 903-995.

Jay, A. D., and E. P. Flinchem, 1999: A comparison of methods for analysis of tidal records containing multi-scale non-tidal background energy. Cont. Shelf Res., 19, 1695-1732.

—_, and T. Kukulka, 2003: Revising the paradigm of tidal analysis-The uses of non-stationary data. Ocean Dyn., 53, 110 125.

Johns, W. E., T. N. Lee, F. A. Schott, R. J. Zantopp, and R. H. Evans, 1990: The North Brazil Current retroflection: Sea- 
sonal structure and eddy variability. J. Geophys. Res., 95 (C12), 22 103-22 120.

Munk, W., and D. Cartwright, 1966: Tidal spectroscopy and prediction. Philos. Trans. Roy. Soc. London, A259, 533-581.

Pawlowicz, R., B. Beardsley, and S. Lentz, 2002: Classical tidal harmonic analysis including error estimates in Matlab using T_TIDE. Comput. Geosci., 28, 929-937.

Pugh, D., 1987: Tides, Surges and Mean Sea-Level. A Handbook for Engineers and Scientists. John Wiley \& Sons, 472 pp.

Santos, M. S., 1999: Descrição de corpos arenosos de grande escala na zona costeira e na plataforma continental norte do Rio Grande do Norte por imagens TM/Landsat. M.S. thesis, Division of Remote Sensing, Instituto Nacional de Pesquisas Espaciais, INPE-7010-TDI/658, $123 \mathrm{pp}$.

Signorini, S. R., and L. B. Miranda, 1983: Tidal and low-frequency currents near the shelf-break: Northeastern coast of Brazil. $J$. Phys. Oceanogr., 13, 2107-2115.

Testa, V., and W. J. Bosence, 1999: Physical and biological controls on the formation of carbonate and silicate bedforms on the northeast Brazilian shelf. Sedimentology, 46, 279-301.

Vianna, M., R. Solewicz, A. Cabral, and V. Testa, 1991: Sandstream on the northeast Brazilian shelf. Cont. Shelf Res., 11, 509-524.

_, A. Cabral, and F. Gherardi, 1993: TM-Landsat imagery applied to the study of the impact of global climate change on a tropical coastal environment during the last deglaciation. Int. J. Remote Sens., 14, 2971-2983.

Vianna, M. L., and V. V. Menezes, 2003: A seasonal and interannual study of the western equatorial Atlantic upper thermocline circulation variability. Interhemispheric Water Exchange in the Atlantic Ocean, G. Goni and P. MalanotteRizolli, Eds., Oceanographic Series, Vol. 68, Elsevier, 137174.

WOCE, 2002: WOCE global data, version 3.0. WOCE International Project Office, Tech. Rep. 180/02, DVD 1 and 2. 